\title{
DESIGN OF MEASUREMENT STRATEGIES FOR WORKPLACE EXPOSURES
}

Hans Kromhout

Occup Environ Med 2002;59:349-354

M easurement strategies for hazard control will have to be efficient and effective to protect a worker's health and well being. No measurement strategy for hazard control will ever be cost efficient in the short run when it is compared with the promises of tools such as the Control of Substances Hazardous to Health (COSHH) essentials (box 1): "a simple system of generic risk assessments which leads to the selection of an appropriate control approach". ${ }^{1}$ Going straight to benchmark standards without the need of exposure measurements will certainly eliminate the cost of measurements. However, generic risk assessment tools like COSHH essentials and expert systems like the Estimation and Assessment of Substances Exposure (EASE) ${ }^{2}$ (box 2), as well as expert judgement by an occupational hygienist, are known to be inaccurate and they do not take into account the various components of variability in exposure levels (box 3). In fig 1, results of EASE estimates are compared with actual measured concentrations. From these pictures it can be seen that EASE estimates tend to be (1) higher than the measured concentrations, and (2) inaccurate especially at lower "true" concentrations $\left(<50 \mathrm{ppm}\right.$ and $<5 \mathrm{mg} / \mathrm{m}^{3}$ ). Nowadays, the latter exposures are being more relevant for workplaces of the developed world.

Proper exposure assessment with the help of measurements will certainly involve short term costs of monitoring. However, ill advised control measures will arguably be even more costly in the long run, a classic case of being "penny wise but pound foolish".

\section{EXPOSURE VARIABILITY}

Correspondence to: Dr H Kromhout, Environmental and Occupational Health Division, Institute for Risk Assessment Sciences, University of Utrecht, PO Box 80176, Yalelaan 2, 3508 TD Utrecht, The Netherlands;

H.Kromhou†@iras.uu.n
Measurements of workplace exposures are crucial not only for evaluations of potential health risks, but also for their subsequent reduction through efficient control measures. When designing measurement strategies the generally large sources of exposure variability should be appreciated in order to design efficient and effective protocols. Exposure concentrations are known to be extremely variable, especially within a day over short averaging times (fig 2 ). On the other hand, long term decreasing trends in exposure concentrations at an average rate of $6 \%$ per annum have recently been described for workplace exposures in western industrialised countries. ${ }^{3}$ Therefore, variability in yearly average concentrations will be much smaller than variability in eight hour shift long measurements that have been estimated to vary between 3-4000 fold. ${ }^{4}$ For 10 second point estimated levels of magnetic field exposure for workers in the utility industry it was estimated that they on average varied an additional 50 fold. ${ }^{5}$ (fig 2 ).

The extent to which exposures vary depends on many factors. ${ }^{4}$ Some of these are concerned with the agent itself, but the majority are linked to work content, tasks performed, production, and environment characteristics. In general, analytical and sampling errors play a minor role in overall exposure variability.

Recently, a database with repeated shift long inhalation exposure measurements from a variety of workplaces and industries was constructed and analysed. For a group of workers (defined by job title and location) it was estimated that personal mean inhalation exposures fall on average within a fivefold range. In a temporal sense, daily exposures were estimated to fall on average within a 15 -fold range. ${ }^{4}$ Even more recently, a similar database was built for dermal exposure and again there was evidence of a larger temporal component than personal component. ${ }^{6}$ Spatial variability (differences in exposure between body locations) was estimated to be even more prominent.

Breakdowns of variance components by industry, production, and environmental factors revealed important differences that should be taken into account when designing measurement strategies (box 4). For example, for outdoor exposures temporal effects tended to outweigh completely minor differences between workers in the same group. For crews of paving workers with distinct jobs this was recently corroborated. Their exposure to bitumen fume appeared to be uniform despite differences in jobs performed. Temporal variability of their exposure was up to a 30 -fold range. ${ }^{7}$ 
Box 1 Control of Substances Hazardous to Health (COSHH) essentials: easy steps to control chemicals

- Generic risk assessment tool aimed at controlling exposure and

meet legal duties by a one stop approach

- Aimed at the employer

- Primarily focused at chemicals supplied for use at work

Not meant for hazards arising from work activities (for example, wood dust, welding fumes, oil mists, etc), chemicals like pesticides and veterinary medicines, naturally occurring and biological hazards, and a few agents such as lead and asbestos with their own regulations

- One stop by going from identifying factors which affect exposure

to control approaches to reduce exposure involving five steps

Four control approaches:

-general ventilation

-engineering control

-containment

-special

Box 2 Estimation and Assessment of Substances Exposure (EASE) model

Expert model developed by Health and Safety Executive (UK)

Based on measured exposure levels from National Exposure

Database (NEDB)

Used to assess workplace exposure for risk assessment of new and existing substances

- Estimates based on exposure scenarios according to use patterns

(4), control patterns (5), and physicochemical properties of substances (6)

- Results in 120 possible combinations that are distilled down to 39

fields for which ranges of concentrations have been assigned

- First validation results show considerable overestimation of

workplace exposure (conservative estimates)

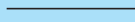

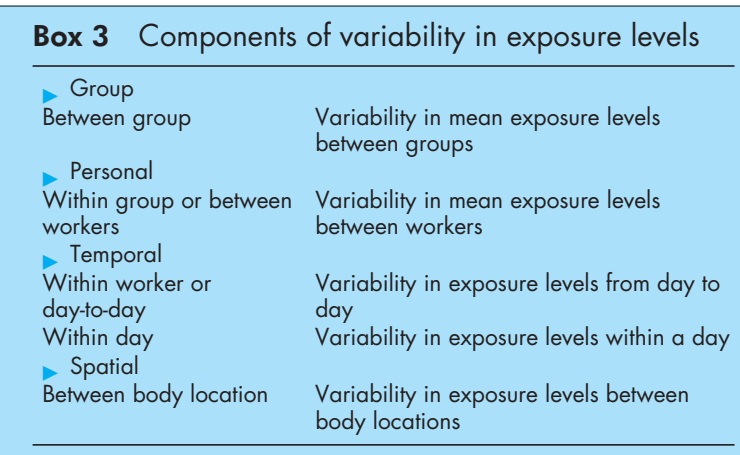

\section{STANDARD MEASUREMENT STRATEGIES FOR COMPLIANCE WITH EIGHT HOUR TIME WEIGHTED AVERAGE LIMIT VALUES}

Traditionally worst case sampling strategies were thought to be very efficient, because they focused measurements upon situations where they were most needed. This approach of sampling the "maximum risk employee" has been widely propagated from the 1970s onwards, primarily as a result of the appearance of the National Institute of Occupational Safety and Health (NIOSH) occupational exposure sampling strategy manual. ${ }^{8}$ Similar considerations also form the basis of the European Norm 689 Guidance for the assessment of exposure to chemical agents for comparison with limit values." However, because of the restrictions typical of these measurement
Box 4 Workplace situations and number of measurements

\begin{tabular}{ll}
\hline More measurements & Fewer measurements \\
\hline Outdoors & Indoors \\
Intermittent process & Continuous process \\
Local exhaust & No local exhaust \\
ventilation & ventilation \\
Mobile worker & Stationary worker \\
Local source & General source \\
Handwork & Highly automated \\
\hline
\end{tabular}

A

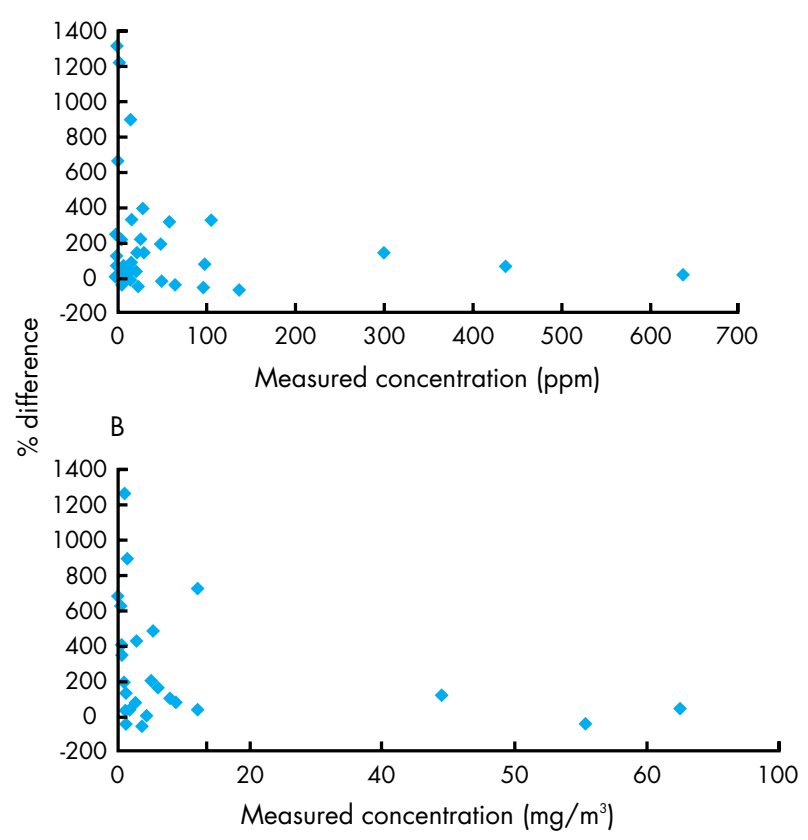

Figure 1 Percentage of difference between geometric mean of EASE estimates and geometric mean of measured concentrations against (A) measured gases/vapours concentrations, and (B) measured dust concentrations. Reproduced from Tijdschrift voor toegepaste Arbowetenschap 2000;13:38-9, with permission of the publisher.

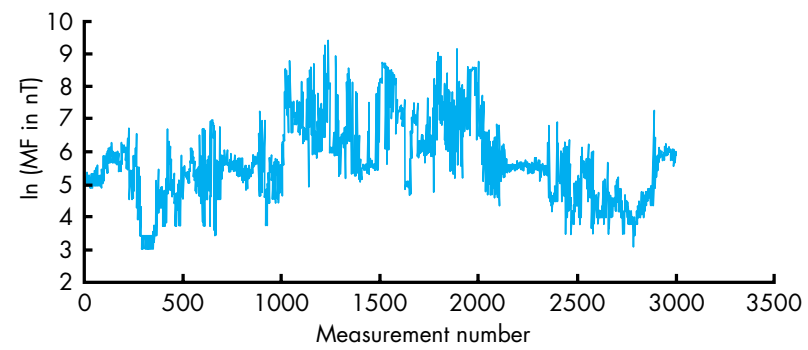

Figure 2 Exposure profile of 10 second interval magnetic fields (logarithms in nano-Tesla) within a shift for a substation electrician.

strategies, they produce incomplete and upwardly biased pictures of exposure concentrations. In addition, these measurement strategies assume implicitly that workers sharing the same environment and tasks are similarly exposed and therefore experience the same risks. Given modern understanding of exposure variability this constitutes a far too rosy and simplistic picture. 
Level 1 Assign observational group
Figure 3 Protocol for assessing and controlling worker specific mean exposures. ${ }^{1013}$

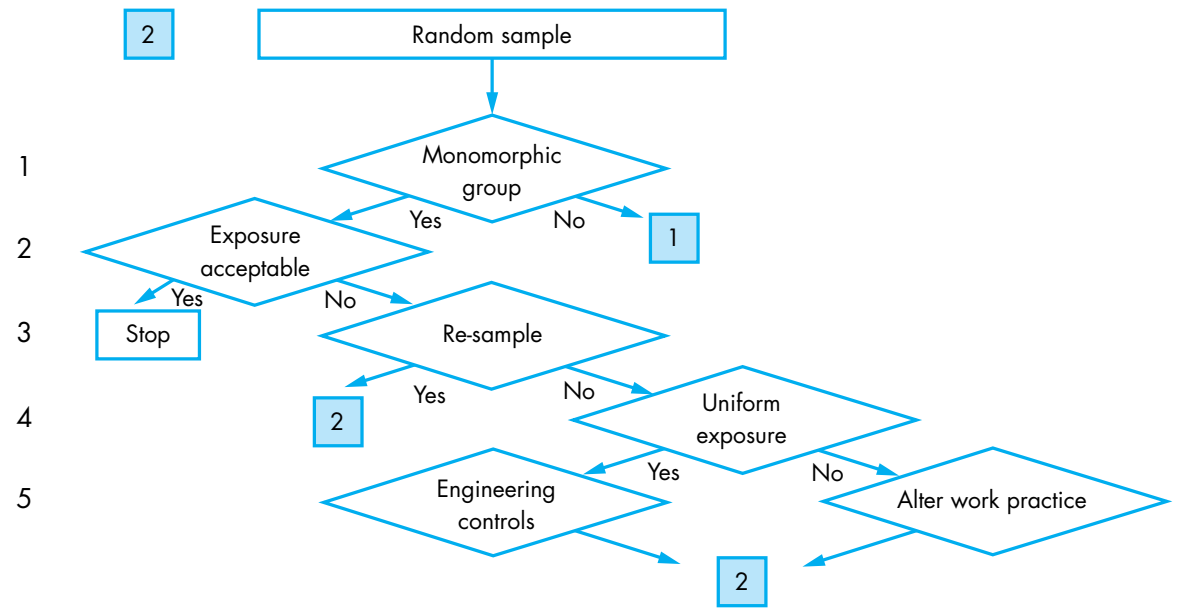

\section{EFFICIENT MEASUREMENT STRATEGY FOR HAZARD CONTROL}

Recently, a new measurement strategy for evaluation of long term average concentrations has been developed that incorporates the notion that workers are not necessarily similarly exposed even though they may work in the same environment and basically perform similar tasks. ${ }^{10}{ }^{11}$ George et al described a striking example of this phenomenon in a car manufacturing plant, where workers were exposed to isopropyl alcohol while wiping an automobile in a heavily ventilated downdraft booth (90 nominal air changes per hour). ${ }^{12}$ Even under these well controlled circumstances there appeared to be an almost fourfold difference in long term average exposure between two workers because of differences in body length, resulting in different distances to the exposure source and consequently different mean exposure concentrations.

In the newly developed measurement strategy, sampling is performed on randomly chosen workers from an a priori identified group of workers (observational group) on repeated days. ${ }^{10}{ }^{11}$ A schematic overview of this measurement protocol is presented in fig 3. After this initial sampling effort the protocol fits a random effects analysis of variance (ANOVA) model to the logged exposure data, and the goodness-of-fit of this model is checked by a graphical procedure. If the fit is acceptable for a given observational group the group is regarded as monomorphic and subsequently tested for overexposure. If the group cannot be considered to be monomorphic an alternative grouping should be attempted. For a monomorphic group the estimated statistical parameters are used to assess whether the probability is acceptably small that a randomly selected worker would be overexposed (that is, a randomly selected worker's mean exposure is above an occupational exposure limit). For groups of workers whose exposure were found to be unacceptable, re-sampling is suggested in some cases to increase the power of the test for declaring existing exposures acceptable. If re-sampling and consequent retesting do not result in an acceptable situation the protocol tests the uniformity of the exposure (that is, whether workers have essentially the same mean exposure) based upon a graphical evaluation of predicted mean exposures for all workers. This last test suggests one of the two types of interventions needed. Interventions are either at individual level (altering of personal environments) or at group level (general engineering controls) for non-uniform and uniform exposed groups, respectively. A computer programme based on this measurement strategy is available as a Microsoft Excel 95/97 application. This computer programme performs all the statistical procedures that are necessary for the described protocol. ${ }^{13}$ It has been suggested that, as a minimum, two samples taken on randomly chosen days of a sample of five workers from an observational group ( 10 samples in total) are needed for this protocol.

\section{EFFICIENT DESIGNS FOR EXPOSURE MEASUREMENTS IN EPIDEMIOLOGICAL STUDIES}

In 1952 Oldham and Roach ${ }^{14}$ described a long term sampling procedure for measuring coal dust exposure among colliers as part of a longitudinal study of pneumoconiosis. The strategic aspects of their "random colliers" method are thought provoking even now. Remarkably, this sampling strategy was implemented without the convenience of portable personal dust samplers. In 1958 Ashford $^{15}$ extended their approach to the "man-shift" method, with which the cumulative coal dust exposure of 35000 colliers from 25 collieries was estimated. For each a priori defined stratum or occupational group (based on occupation, place of work and shift) a random selection was made from the population of all man shifts worked by the members of the stratum. The number of measurements allocated to each stratum was proportional to the product of the duration, the standard deviation of the shift exposure indices, the square root of the average number of workers, and a factor representing labour turnover and attendance.

It took more than 40 years to see such an effective measurement strategy being repeated and improved upon. In 1994 Loomis et al constructed occupational categories to organise thousands of job titles at five electric utility companies participating in a cohort mortality study. ${ }^{16}$ The 28 categories were aggregated into three ordinal levels of presumed magnetic field exposure. A goal of 4000 full shift magnetic field measurements was set, based principally on considerations of time, cost, and tolerance of the participating companies. The number of measurements to be made in each occupational category was a function of the projected total number of measurements, arbitrary weights of 1,3 , or 5 for the 
three exposure levels, and a second set of weights proportional to person-years of employed experience contributed by each of the five companies. The rationale for the weights of 1,3 , and 5 was that groups with higher average exposure would also experience more variable exposures, requiring more measurements to obtain equally precise estimates of average exposures. In order to estimate between and within individual variability in exposure levels, each individual selected in the medium and high exposure groups was measured on two randomly selected days less than 12 months apart.

By using a small integrating personal magnetic field exposure meter, workers and management personnel could conduct the exposure survey in the field, without direct supervision by an occupational hygienist. Meters were distributed to the workers by company mail. A quality assurance scheme was in place to test correct functioning and calibration regularly. Self monitoring of exposure was used to ensure financial feasibility of such an extensive measurement scheme. This scheme resulted in almost 3000 successful measurements that provided objective and statistically sound estimates of cumulative magnetic field exposure for a geographically widely distributed population of utility workers. ${ }^{17}$ Such carefully designed exposure measurement, exposure assessment, and exposure assignment strategies have been shown to be of critical importance to unveil exposure-response relations. ${ }^{18}$ Equations to assist in this decision making process have recently been developed for group based approaches and are well established for individual based approaches. ${ }^{19} 20$

\section{MULTIPURPOSE MEASUREMENT STRATEGIES}

Measurements carried out to assess the likelihood of overexposure for hazard control or to estimate long term average exposures for an epidemiological study could have uses beyond those originally envisioned. A prerequisite for this is that contextual information be collected and recorded in a standard way about tasks, types of processes, and presence of control measures which were present during monitoring. The first empirical statistical models were built on this type of information in the early 1980s. ${ }^{21}{ }^{22}$ Statistical models can be used to identify important factors that affect exposure concentrations, as described for several agricultural settings and industries. For an extensive overview of methodological issues in such studies, see the article by Burstyn and Teschke. ${ }^{23}$ Recently, these models have been refined to take into account the lack of independence of repeated measurements of the same worker. ${ }^{24-27}$ A drawback of such models are the large amounts of data needed to develop meaningful and insightful empirical models. With proper exposure assessments under threat (being replaced by expert systems and one stop approaches) the prospects are not very promising. However, Burstyn et al showed that even with data of varying quality from different countries a large database of asphalt workers' exposure could be constructed, from which determinants of exposure and long term trends in exposure could be distilled. ${ }^{27}$ These were consequently used to develop exposure matrices for a large multicentre cohort study. Remarkably 33 out of the 37 datasets present in the international database were never described in the open literature.

Identified factors affecting exposure can consequently be used to lower exposure levels (a continuous improvement strategy). A prospective study in the rubber manufacturing industry in The Netherlands showed the effectiveness of such an approach. ${ }^{26}$ Control measures taken in this longitudinal study were, to a certain extent, based on originally identified exposure affecting factors. The study showed that elimination of sources and control measures designed to control the levels of contaminants were highly effective and resulted in a $40-50 \%$ reduction of both inhalation and dermal exposure over a nine year period.

\section{TASK BASED MEASUREMENT STRATEGIES AND AN ALTERNATIVE}

A totally different approach is the task based approach where a priori assumptions are explicitly made on tasks leading to presumed high(er) exposures and workers are only monitored when performing tasks under consideration. Risk assessors favour this approach especially for pesticide registration purposes. Measurement data for these purposes are collected for tasks performed under controlled conditions, exemplifying "best practice" advocated by chemical producers. ${ }^{28}$ Even though this approach might be reasonable for registration purposes (risk assessment) it is doubtful that exposure data collected in this manner can be used beyond the original goal. They certainly cannot be used to predict exposure levels under realistic field circumstances. Such data can be expected to suffer from the same shortcomings as traditional worst case strategies, only being their mirror image: "best case" sampling. In addition, using task based sampling data to derive long term average exposure measures requires collection of detailed information on duration of tasks. This information is known to be unreliable especially when collected retrospectively. However, it has been argued that in modern times exposure more often occurs intermittently because of practices such as job rotation (for example, exposure to stainless steel welding fumes among welders in a shipyard occurring only for one hour once a week). Monitoring a worker's exposure for a complete work shift on a randomly chosen day under such circumstances might result in non-detectable levels and/or underestimation of exposure levels. Non-random task-based sampling might be more efficient in this case.

Alternatively, it has been shown that in exposure conditions with infrequent occurring tasks (with high exposures) full shift random sampling could be applied effectively as long as simultaneously daily activities are being registered. ${ }^{29}$ Based on measurement data from a large number of individuals and information on daily activities during the measurements, one can construct empirical statistical models that will unravel exposure affecting factors. These models can consequently be used to predict long term average exposure based on information on daily activities collected for a longer period than during which samples were collected. Recently, it was theoretically proven that, given the same monitoring effort, this approach leads to more accurate estimates of long term exposure than those based solely on measurements. ${ }^{30}$ In practice, of course, this approach will stand or fall on the availability of contextual information (box 5) such as information on daily activities and production characteristics.

In conclusion, non-random task based measurements will by their nature have a limited scope. Nevertheless they can be used to estimate exposures occurring very infrequently. A combination of full shift random measurement strategies with additional task based measurements for exposure conditions likely to be missed in a random measurement strategy will be the preferred approach in those situations.

\section{CONCLUSION}

Effective measurement strategies will have to result in unbiased estimates of worker's exposure, but should at the 
Box 5 Contextual information to be collected and registered during measurements

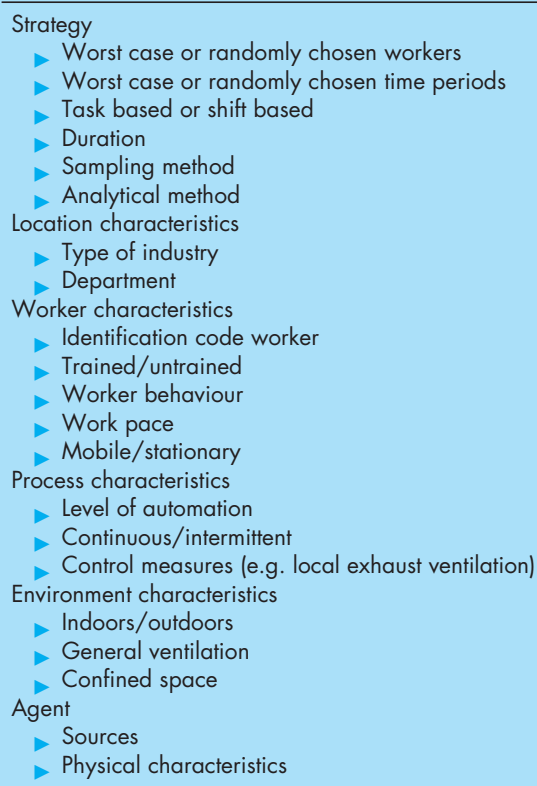

same time collect enough detailed contextual information to facilitate well advised control measures. Their efficiency will depend on the possibilities for limiting the input of occupational health professionals in the collection of data. On the other hand, using costly measurements for multiple purposes (hazard evaluation, control, and epidemiology) will make them more cost efficient as well.

In order to design efficient and effective measurement strategies for workplace exposures we need to have insight into the magnitude of variability in a spatial sense (body locations when dealing with dermal exposure), as well as a temporal (day-to-day), personal (between workers), and group (between groups) sense. Realistically, budgets for measurements of workplace exposures will never allow the amount of resolution that will enable us to reconstruct exposure for each individual worker on any given day of his or her working life. The recent elaboration and consequent statistical analysis of large databases with repeated measurements has provided us with insights into exposure variability that will help us to design more efficient and effective surveys to measure long term average exposure. New measurement strategies incorporating this knowledge have been developed as a result. Old and new simplistic approaches entirely relying on deterministic exposure models (expert systems) or subjectively estimating experts cannot replace proper quantitative exposure assessment, if our overall goal is to protect workers' health rather than to meet short term budgetary goals. Therefore, denying the presence of extensive sources of variability in workplace exposure concentrations is clearly unscientific. Such denial ignores the fact that some workers, clearly differently exposed than their fellow workers, will be deprived of necessary controls. On the other hand, cutting the costs of extensive measurement surveys by involving workers in the sampling procedure (self assessment) and making better use of existing data has been shown to be effective in the short and long run. In a recent paper by Liljelind and colleagues it appeared that untrained, unsupervised workers were able to collect unbiased exposure data (on gaseous contaminants) by using currently available passive monitors. ${ }^{31}$

A prerequisite for such approaches is of course availability and development of (1) relatively simple measurement devices (that is, passive monitoring badges), (2) comprehensive exposure databases, and (3) easy tools to handle the increasingly complex statistical analyses.

Occupational hygienists and other occupational health professionals reconsidering whether measurement has to be their primary tool should have a look at the referenced literature. The new findings and approaches propagated for efficient and effective measurement will hopefully convince them that measurements are still needed and feasible.

\section{ACKNOWLEDGEMENTS}

I would like to acknowledge Igor Burstyn, Dana Loomis, Steve Rappaport and Roel Vermeulen for providing me with the fruits of our collaboration over the years, without which the contents of this paper would have been quite different.

\section{REFERENCES}

1 Topping M. Occupational exposure limits for chemicals. Occup Environ Med 2001;58:138-44

2 Friar JJ. The assessment of workplace exposure to substances hazardous to health - the EASE model. Draft guidance paper, Health \& Safety Executive, 1996.

3 Kromhout $\mathbf{H}$, Vermeulen R. Long-term trends in occupational exposure: Are they real? What causes them? What shall we do with them? Ann Occup Hyg 2000;44:325-7.

4 Kromhout H, Symanski E, Rappaport SM. A comprehensive evaluation of within- and between-worker components of occupational exposure to chemical agents. Ann Occup Hyg 1993;37:253-70.

- The first comprehensive evaluation of temporal and personal variability in exposure concentrations. A database of approximately 20000 chemical exposures predominantly from the Netherlands and the USA was constructed. Most observational groups of workers appeared to be not uniformly exposed. Environmental and production factors were shown to have distinct influences on the day-to-day variability, but hardly no influence on the between-worker variability.

5 van der Woord MP, Kromhout $\mathrm{H}$, Barregård L, et al. Within-day variability of magnetic fields among electric utility workers: consequences for measurement strategies. Am Ind Hyg Assoc J 2000;61:31-8.

6 Kromhout $H$, Vermeulen R. Temporal, personal and spatial variability in dermal exposure. Ann Occup Hyg 2001;45:257-73.

- Description of components of variability in dermal exposure data on the basis of a large database with more than 6000 measurements from 20 surveys.

7 Burstyn I, Kromhout H. Are the members of a paving crew uniformly exposed to bitumen fume, organic vapour and benzo(a)pyrene? Risk Analysis 2000;20:653-63.

8 Leidel NA, Busch KA, Lynch JJ. Occupational exposure sampling strategy. Cincinnati, Ohio: National Institute for Occupational Safety and Health, DHEW (NIOSH) publication number 77-173, 1977

- The manual that explained intent and purposes of employee monitoring requirements for employers in the USA.

Recommendations were given to meet the legal requirements with minimum burden to the employer. It provides data analysis methods to assist in making better decisions. Valuable but somewhat dated reference manual.

9 CEN. Workplace atmospheres. Guidance for the assessment for exposure by inhalation to chemical agents for comparison with limit values and measurement strategy. Brussels: CEN, EN 689, 1995

- The current EU standard that provides a framework for the assessment for exposure to chemical agents for comparison with limit values. The standard provides no measurement strategy to be followed and leaves this open to professional judgement. Some examples of approaches and statistical procedures can be found in its annexes.

10 Rappaport SM, Lyles RH, Kupper LL. An exposure-assessment strategy accounting for within- and between-worker sources of variability. Ann Occup Hyg 1995;39:469-95.

- Theoretical background of a new measurement strategy that accounts for components of variability that have been shown to be prevalent in the work environment of groups of workers. With illustrative examples based on exposures in the nickel industry.

11 Lyles RH, Kupper LL, Rappaport SM. A lognormal distribution-based exposure assessment method for unbalanced data. Ann Occup Hyg 1997;41:63-76.

Extension of the proposed measurement strategy described in reference 10 to situations in which not all individuals have been sampled on the same number of occasions. 
12 George DK, Flynn MR, Harris RH. Autocorrelation of interday exposures at an automobile assembly plant. Am Ind Hyg Assoc J 1995;56: 1 187-94.

13 Vermeulen R, Kromhout H, Braun W, et al. Statistical program for the evaluation of exposure data. Environmental and occupational health group, Wageningen Agricultural University, 1998.

14 Oldham PD, Roach SA. A sampling procedure for measuring industrial dust exposure. Br J Ind Med 1952;9:112-9.

- The procedure described in this paper should be considered to be the mother of all measurement strategies.

35415 Ashford JR. The design of a long-term sampling programme to measure the hazard associated with an industrial environment. J $R$ Stat Soc Series A 1958;3:333-47.

- Any epidemiologist considering collecting quantitative exposure data for an epidemiological study should start with this classic paper, after which the designed measurement strategy will be considerably more efficient and effective.

16 Loomis DP, Peipins LA, Browning SR, et al. Sampling design and field methods of a large, randomized, multi-site survey of occupational magnetic field exposure. Appl Occup Environ Hyg 1994;9:49-52.

- Extensive description of a large survey of magnetic field exposure among utility workers from five companies throughout the USA for an epidemiological study. The survey used a random measuremen strategy and was facilitated by self assessment by workers.

17 Kromhout H, Loomis DP, Mihlan GJ, et al. Assessment and grouping of occupational magnetic field exposure in five electric utility companies. Scand J Work Environ Health 1995;21:43-50.

18 Kromhout H, Loomis DP, Kleckner RC. Uncertainty in the relation between exposure to magnetic fields and brain cancer due to assessment and assignment of exposure and analytical methods in dose-response modelling. Ann N Y Acad Sci 1999:895:141-55.

19 Tielemans E, Kupper LL, Kromhout H. et al. Individual-based and group-based occupational exposure assessment: some equations to evaluate different strategies. Ann Occup Hyg 1998;42: 115-9.

- Theoretical paper giving insight into the consequences of choosing individual or group based approaches in the collection and analyses of quantitative exposure data. Equations are given that will facilitate data driven decisions for optimal exposure measurement and assessment.

20 Liu K, Stamler J, Dyer A, et al. Statistical methods to assess and minimize the role of intra-individual variability in obscuring the relationship between dietary lipids and serum cholesterol. J Chron Dis 1978;31:399-418.

21 Eisen EA, Smith TJ, Wegman DH, et al. Estimation of long term dust exposures in the Vermont granite sheds. Am Ind Hyg Assoc J 1984;45:89-94.

22 Dement JM Harris RL, Symons M, et al. Exposure and mortality among chrysotile asbestos workers. Part 1: exposure estimates. Am J Ind Med 1983;4:399-419

23 Burstyn I, Teschke K. Studying the determinants of exposure: a review of methods. Am Ind Hyg Assoc J 1999;60:57-72.

24 Nylander-French LA, Kupper LL, Rappaport SM. An investigation of factors contributing to styrene and styrene-7,8-oxide exposures in the reinforced-plastics industry. Ann Occup Hyg 1999;43:99-105.

25 Rappaport SM, Weaver M, Taylor D, et al. Application of mixed models to assess exposures monitored by construction workers during hot processes. Ann Occup Hyg 1999:43:457-69.

26 Vermeulen R, de Hartog J, Swuste P, et al. Trends in exposure to inhalable particulate and dermal contamination in the rubber manufacturing industry: effectiveness of control measures implemented over a nine-year period. Ann Occup Hyg 2000;44:343-54.

27 Burstyn I, Kromhout H, Kauppinen T, et al. Statistical modelling of the determinants of historical exposure to bitumen and polycyclic aromatic hydrocarbons among paving workers. Ann Occup Hyg 2000;44:43-56.

28 van Hemmen JJ. EUROPOEM, a predictive occupational exposure database for registration purposes of pesticides. Appl Occup Environ Hyg $2001 ; 16: 246-50$

29 Preller L, Kromhout $H$, Heederik D, et al. Modelling long-term average exposure in occupational exposure-response analysis. Scand J Work Environ Health 1995;21:504-12.

30 Wameling $\mathbf{A}$, Schäper $M$, Kunert J, et al. Individual tolvene exposure in rotary printing: increasing accuracy of estimation by linear models based on protocols of daily activity and other measures. Biometrics 2000;56:1218-21.

31 Lilielind IE, Rappaport SM, Levin JO, et al. Comparison of self-assessment and expert assessment of occupational exposure to chemicals. Scand J Work Environ Health 2001;27:311-17.

\section{QUESTIONS (SEE ANSWERS ON P 286)}

(1) Which of the following sources is expected to affect exposure variability the most when measurements are being conducted with a device with an averaging time of one minute for several days in two groups of workers performing the same tasks at two different locations?

- (a) within worker (day-to-day) variability

- (b) within day variability

- (c) between worker (within group) variability

- (d) between group variability

(2) When performing measurements of workplace exposures for epidemiological studies efficiency can be gained by:

- (a) restricting measurements to the highest exposed workers

- (b) by measuring on one occasion only

- (c) by self-assessment

- (d) by collecting contextual information

(3) Present day expert systems like EASE can replace proper exposure assessment:

- (a) yes, since EASE is of a deterministic nature and based on physical properties

- (b) yes, EASE is accurate enough to evaluate individual risks

- (c) no, EASE should only be used as a method for initial appraisal

- (d) yes, EASE takes into account spatial, temporal and personal variability

(4) Repeated full shift sampling of the same worker is necessary:

- (a) to be able to estimate temporal variability in daily concentrations

- (b) to be able to estimate between worker variability in mean exposure concentrations

- (c) to be able to estimate both within and between worker variability in exposure concentrations

- (d) to be able to estimate spatial variability in exposure concentrations

(5) In a plant, workers are assigned to observational groups, and individuals and days to be monitored are randomly chosen. Some workers are scheduled to be measured on more than one occasion. Contextual data are collected in a standardised manner and recorded along with the results of the measurements. Which of the following will be possible after the data have been collected:

- (a) calculation of each individual's mean exposure

- (b) identification of factors affecting exposure

- (c) estimating temporal and personal variability in exposure concentrations

- (d) all of the above 